\title{
Dynamic Reflection for a Statically Typed Language
}

\author{
Søren Brandt and René W. Schmidt \\ Department of Computer Science \\ University of Aarhus \\ DK-8000 Aarhus C, Denmark
}

\begin{abstract}
We present a runtime metalevel interface for BETA. BETA is a compiled and statically typed object-oriented programming language. The metalevel interface preserves the type safe properties of the language and supports static type checking. This is achieved through a novel language construct, the attribute reference, on top of which the metalevel interface is built. The metalevel interface is based on a simple conceptual model that reifies a few basic language primitives. For the implementation, a metalevel architecture based on a virtual machine view of the runtime system is introduced. In this model, an open implementation of compiled language is achieved by providing the runtime virtual machine with a metalevel interface supporting runtime reflection.
\end{abstract}

\section{Introduction}

Reusability is a main ingredient in most scientific and technical disciplines. Scientific theories are building blocks for a deeper understanding of various systems, well-engineered technical devices serve as building blocks for larger systems, and software components should in general be able to form part of multiple end-user applications. The keywords are modularity, extensibility, and tailorability.

Structured programming turned modularity into the single most important program design principle. Extensibility and tailorability are major reasons for the recent success of object-oriented programming, which allows the programmer to treat new classes as built-ins, and tailor existing classes through subclassing and similar mechanisms.

However, while object-oriented programming languages provide excellent facilities for expressing abstractions in many application domains, most languages fall short in the disciplines of extensibility and tailorability of their own domain. 


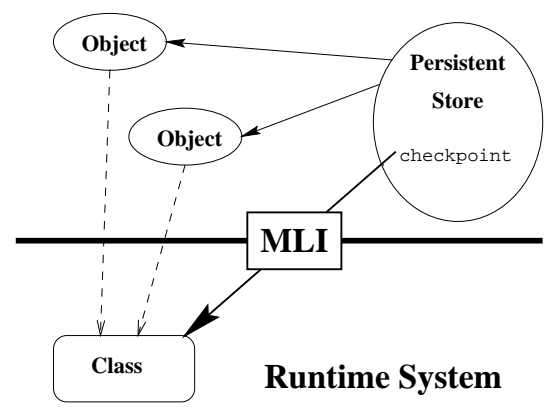

Figure 1: A Persistent Store using runtime metalevel information.

Extension of a programming language with new type-orthogonal functionality such as persistent storage, generic object copying, or distribution is typically not possible. Common for these functionalities are that they all depend on metainformation and possibly implementation details of the language. To circumvent language limitations, the programmer is often forced to use source code preprocessing, special compilers, or new runtime systems.

This paper presents MetaBETA, an open implementation [Kiczales 92] of the BETA language [Madsen et al. 93]. By providing a metalevel interface, MetaBETA extends the BETA language with support for writing abstractions for the language directly in the language.

The metalevel interface (MLI) is an abstract interface to the implementation of a running program. Through the introspective part of the MLI, programs can get an abstract description of the layout of any object. Through the intercessory part, basic language primitives such as object invocation, creation, and destruction can be changed. Figure 1 depicts a persistent store utilizing the introspective interface to access type information, thereby being able to serialize arbitrary objects to stable storage. In MetaBETA, the metalevel interface is runtime based, so the persistent store can serialize any object, independent of its class and creator. Access to source code is not necessary.

BETA is a statically typed ${ }^{1}$ and compiled language. It is designed for programming-in-the-large, with explicit type annotations to make source code easier to read, maintain, and debug. Thus, adding a MLI raises two major concerns: i) seamless integration of the MLI with the existing type system, and ii) performance retention.

Our approach to handling these concerns is outlined in the next section, where we present the MetaBETA conceptual model and its motivation. Section 3 presents the metalevel interface. Section 4 introduces the metalevel architecture, which is a runtime organization of a compiled program that supports the met-

\footnotetext{
${ }^{1}$ With statically typed we mean that most type checks are done at compile time. As is the case with, e.g., Eiffel [Meyer 92] and Java [Arnold \& Gosling 96], only partial compile-time checking is possible, because subclasses may strengthen the type of inherited attributes. I.e., BETA allows covariant class hierarchies.
} 
alevel interface. Section 5 describes the current status of the project along with preliminary performance results. Section 6 summarizes related work. Finally, Section 7 presents our conclusions.

\section{Design and Motivation}

Th lack of extensibility and tailorability of the current BETA implementation was recognized during the design and implementation of a number of substrate systems for the Mjølner BETA system, including: type-orthogonal persistence [Brandt 94, Grønbæk et al. 94], distributed object system [Brandt \& Madsen 94], an embedded interpreter [Malhotra 93], a source-level debugger, and object- and class-browsers.

These tools manipulate objects in a type-independent manner, and currently depend on the memory layout of objects, as well as other implementation details. Such dependencies severely compromises portability and type-safety. A common trait of these tools is that they all work at the metalevel, using parts of a running BETA program as data. Our work on MetaBETA is aimed at identifying the core metalevel functionality needed by these systems, and to support those needs through a type-safe metalevel interface.

Example 1 A type-orthogonal library such as object distribution must be applicable to any class, even if that class was written without distribution in mind, and even if the source code is not available. Hence, the library must be able to handle object in a type generic fashion.

The design criterias for the metalevel interface was that it should be simple, dynamic, and efficient. By simple, we mean an interface that is easy to use and understand, i.e., well integrated into the existing language. Albeit the metalevel is to be used only for special purposes, it should be a tool for all programmers, and not limited to "kernel-hackers". By dynamic, we mean that metalevel computation is a property that can be added to individual objects or sets of objects at runtime. By efficient, we mean that the performance impact of using the MLI must not be prohibitive, and the overhead of reflectional capabilities should only be paid on use.

Our MLI builds on two concepts: first-class type information and runtime events.

The introspective part of the MLI provides a way to obtain runtime access to class-definitions and type-annotations as they existed in the source code. Typeinformation is reified by pattern references and attribute references. In BETA, patterns $^{2}$ are already first-class values, while attribute references are introduced in MetaBETA to turn attributes ${ }^{3}$ into first-class values as well. Attribute references

\footnotetext{
${ }^{2}$ Similar to a Class in this context. Patterns are described in Section 3.

${ }^{3}$ Similar to slots in CLOS terminology.
} 


\section{Baselevel:}

invocation

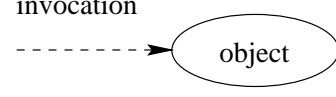

\section{Metalevel:}

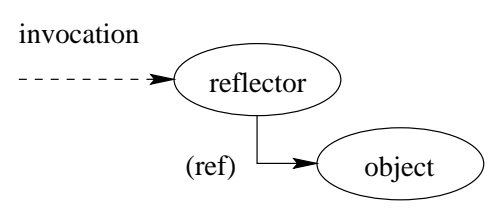

\section{Metametalevel:}

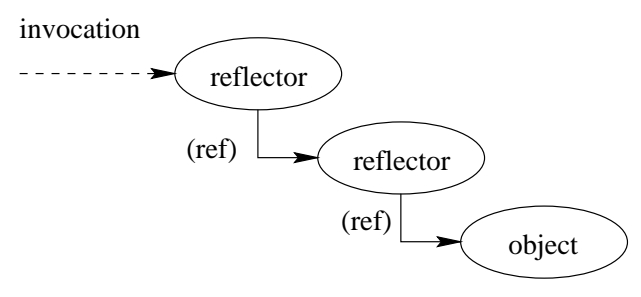

Figure 2: Reflectors and Metalevels.

are the only syntactical difference between BETA and MetaBETA, and integrate seamlessly into the existing type system.

To expose implementation primitives, the intercessory part of the MLI uses reflectors. A reflector is an ordinary programmer-defined BETA object that can be hooked into the runtime system to be automatically invoked whenever a given event occurs. MetaBETA defines three different events, associated with the language primitives object creation, object invocation, and object reclamation. Instead of having a metaobject that controls the execution of each object, there exist a metaobject for each reified language primitive. Thus, reflection can be performed on just parts of an object, making it easier to structure metacode and to control the performance overhead.

Figure 2 despicts the use of reflectors to control object invocation: Normally (baselevel), an object is invoked directly. If a reflector is associated with the invocation-event for a given object, the reflector is invoked instead of the object itself. The reflector is responsible for invoking the object, but is not required to do so. Because a reflector is an ordinary BETA object, it is possible to associate a reflector with a reflector-invocation-event, allowing, in effect, metametalevel computation and so on.

\section{The Metalevel Interface}

This section describes the set of primitives reified by the MetaBETA MLI. MetaBETA programs access the MLI through the globally visible MLI object. 
Attribute references and pattern references are used as the foundation of the metalevel interface, and are described in Sections 3.1 and 3.2. The MLI itself is presented in Sections 3.3 and 3.4.

\subsection{Patterns and Pattern References}

BETA source code is a collection of patterns. The pattern is a unifying abstraction for concepts such as class, method, virtual method, exception, coroutine, and generics. A pattern $\mathrm{P}$ is declared as follows:

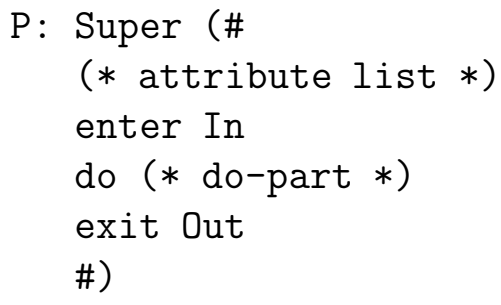

where Super defines an optional superpattern for P, In is an optional enter-list (formal parameters), and Out is an optional exit-list (return values). The do-part of a pattern consists of imperative code executed by instances of $\mathrm{P}$.

Example 2 Figure 3 shows how patterns are used to model both classes and methods. The Calc pattern is used as a class. It has a single nested pattern add which is used as a method. There is no syntactical difference between class patterns and method patterns. The only difference is their usage. Figure 3 also declares an instance, aCalc, of the Calc pattern, and calls its add method. The return value from add is assigned to the integer variable res. Note, that the BETA assignment operator, $->$, assigns from left to right.

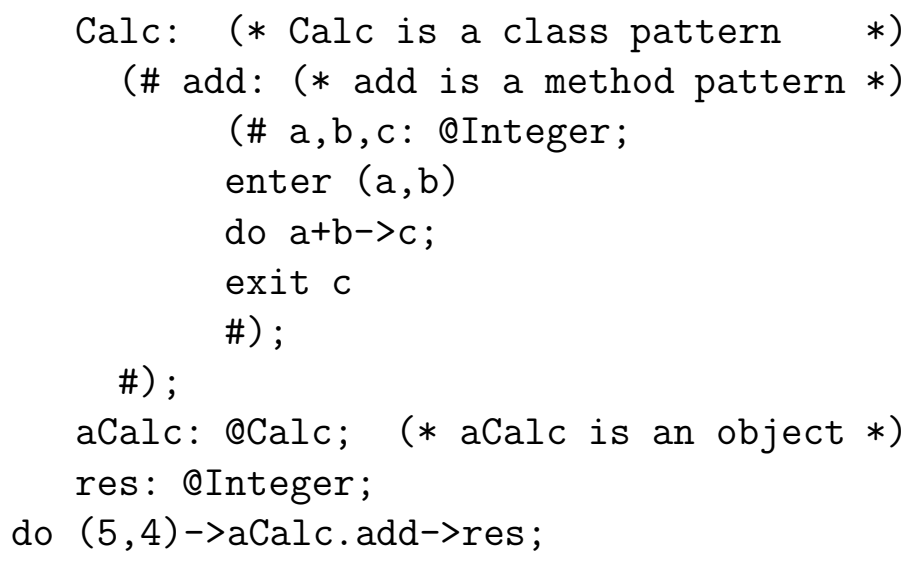

Figure 3: The Calc pattern.

Dynamic pattern references turn patterns into first-class values. Given a dy- 
namic pattern reference, it is possible to create instances of the referred pattern, and to check subpattern relationships by comparing it with another pattern reference. The code below declares a dynamic pattern reference, calcP, assigns the Calc pattern to calcP, and finally creates an instance of Calc:

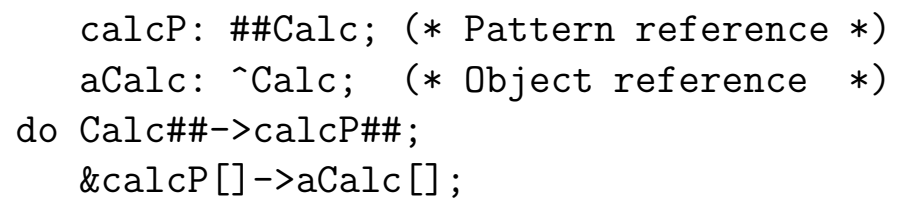

A pattern reference implicitly denotes the set of objects that are instances of the referred pattern (the extension set), and may therefore be used to denote the set of objects a given metalevel operation operates on ${ }^{4}$.

\subsection{Attribute References}

Dynamic pattern references allow programs to explicitly talk about patterns. The MetaBETA MLI also needs to explicitly talk about attributes of patterns. For this purpose we add a new language concept, the attribute reference, to the BETA language.

Attribute reference declarations are typed to allow the compiler and programmer to make assumptions concerning the kind of attribute referred by a particular attribute reference. Metalevel code thereby becomes more readable, and the compiler will be able to generate efficient, type-safe code. An attribute reference is declared as:

\section{attr_ref: .loc_qua*attr_qua}

The syntax includes three qualifications:

1. loc_qua, the (optional) location qualification. The attribute referred by attr_ref must be an attribute of the pattern loc_qua, or of some superpattern of loc_qua.

2. The kind qualification, shown as * in the declaration of attr_ref, constrains the attribute reference to refer specific kinds of attributes. Examples of attribute kinds are dynamic (^) and static (@) object references.

3. attr_qua, the (optional) attribute qualification, constrains the qualification of the attribute referred by attr_ref. For example, an attribute reference

\footnotetext{
${ }^{4}$ To allow pattern references that denote more general extension sets than those corresponding to pattern extensions, the MetaBETA pattern reference mechanism has been generalized into a type reference mechanism, as described in [Brandt \& Knudsen 96]. The generalized mechanism allows pattern references with single-element extension, and pattern references corresponding to sets of patterns.
} 


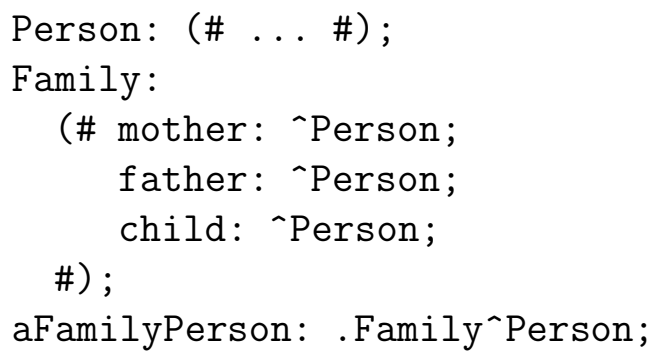

Figure 4: Example of an Attribute Reference.

may be qualified to refer only attributes referring text objects.

Example 3 The MetaBETA fragment in Figure 4 declares the patterns Person and Family, and the attribute reference aFamilyPerson. Families have attributes mother, father, and child, each of which are dynamic references to person objects. The attribute reference aFamilyPerson has location qualification Family, kind qualification "dynamic object reference", and attribute qualification Person, which qualifies it to refer any of the family attributes. The attribute reference can be used as:

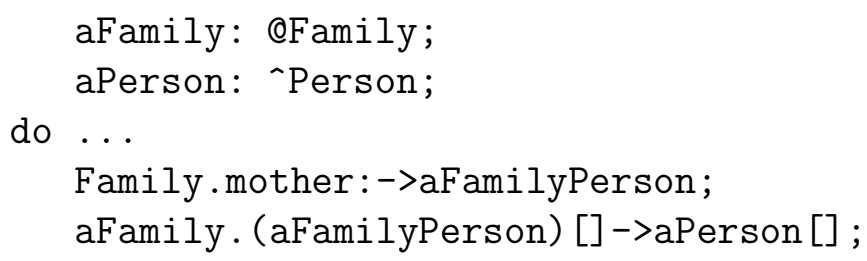

First, the Family.mother attribute is assigned to the aFamilyPerson attribute reference. Then, aFamilyPerson is applied to the aFamily object, thus returning a reference to the mother in aFamily. In this example, all necessary type-checking can be done at compile-time due to attribute reference qualifications.

Using the syntax:

uc_attr_ref : .?

we may declare an unconstrained attribute reference that is allowed to refer any kind of attribute, including attribute reference attributes themselves.

\subsection{Introspective Capabilities}

An executing program can access metainformation about itself by using the introspective capabilities. Metainformation is represented as pattern and attribute references. The MLI allows extraction of complete object descriptions modulo the do-parts. 
Introspecting Attribute References Given an attribute reference, the BETA MLI allows extraction of the name of the attribute, the qualification of the attribute, and the attribute kind. Qualifications are returned as dynamic pattern references that, e.g., can be compared against known patterns to locate attributes of specific types in an object.

Introspecting Pattern References Given a pattern reference, $p$, it is possible to scan the attributes that exist in instances of $p$. For each attribute of $p$, an attribute reference, ar, is returned. Persistent stores, object browsers, and schema evolution tools, to mention a few, can use this to handle objects in a type independent manner.

The scanDyn method of the MLI provides a way to scan all object reference attributes of a pattern:

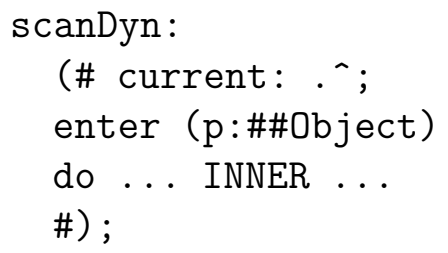

The scanDyn iterator pattern calls INNER for each attribute of the pattern $\mathrm{p}^{5}$.

Example 4 To print the names of all dynamic object reference attributes of the Family pattern, we can write:

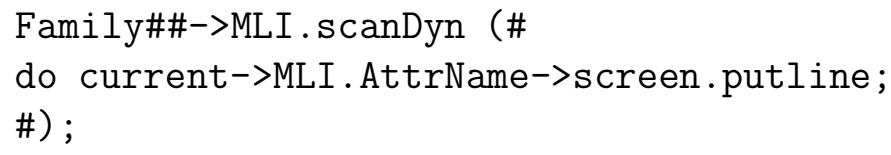

This will print out the text strings mother, father, and child. The real MLI allows subpatterns of scanDyn to strengthen the qualification of current, thus supporting static type-checking of code inside subpatterns of scanDyn that use current to access object attributes.

\subsection{Intercessory Capabilities}

The intercessory capabilities opens up the runtime system, providing access to the implementation of language primitives. The MLI provides an abstract interface to the implementation of object creation, object execution, and object destruction.

The MLI presents each reified primitive by: i) a controller object in the runtime system; ii) a reflector pattern, which is used as superpattern for user-defined

\footnotetext{
${ }^{5}$ Execution of a method $m$ always starts at the least-specific specialization of $m$, and continues down the specialization-chain at each INNER imperative. An INNER imperative for which no specialization is specified corresponds to the empty imperative.
} 
reflectors; iii) a method that directly executes the reified primitive; and iv) a method that invokes the default implementation of the primitive, shortcutting the reflectional mechanisms. Table 1 summarizes the intercessory mechanisms supported by the MetaBETA MLI. These mechanisms are described in detail in the following sections.

\begin{tabular}{|l||l|l|c|c|}
\hline Primitive & Controller & Reflector & Callable Int. & Default Impl. Int. \\
\hline \hline Obj. Creation & AllocCntr & AllocReflector & $\&$ & defaultAlloc \\
\hline Obj. Execution & ExecCntr & ExecReflector & exec & defaultExec \\
\hline Obj. Deletion & GCCntr & GCReflector & N/A & N/A \\
\hline
\end{tabular}

Table 1: MetaBETA Intercession Primitives.

\subsubsection{Object Creation}

A new BETA object is created with the \& operator. In MetaBETA, \& is reified, allowing user-defined code to be executed in response to \& invocations. The userdefined code is presented to the metalevel interface in the form of instances of the AllocReflector pattern:

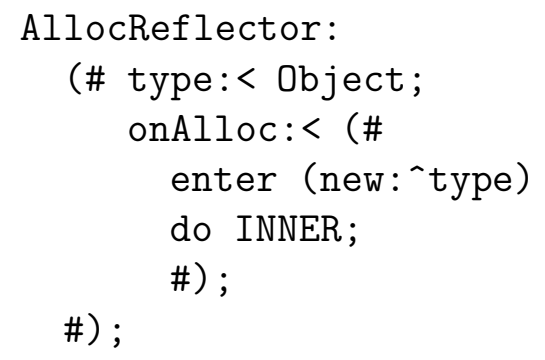

Invocation of AllocReflector objects is controlled by the AllocCntr metaobject whose interface is shown in Figure 5. An AllocReflector is inserted into the runtime system by the AllocCntr.register method. As a parameter, AllocCntr.register takes a pattern reference q, and an AllocReflector reference ar. The q parameter determines the object creations that should trigger the ar.onAlloc virtual ${ }^{6}$ : When objects of type $q$ is created, ar.onAlloc is called with the new object as parameter.

In case several reflectors apply to an object creation, those registered with the most general $\mathrm{q}$ are executed first. If several AllocReflectors are registered with the same q, they are called in last-registered-first-called order.

Example 5 In Figure 6, the reflectors VehicleAR and CarAR are registered to reflect on, respectivly, the allocation of Vehicle and Car objects. Then, a Vehicle

\footnotetext{
${ }^{6}$ onAlloc is a virtual pattern, as determined by $:<$, and can be specialized in subpatterns of AllocReflector.
} 


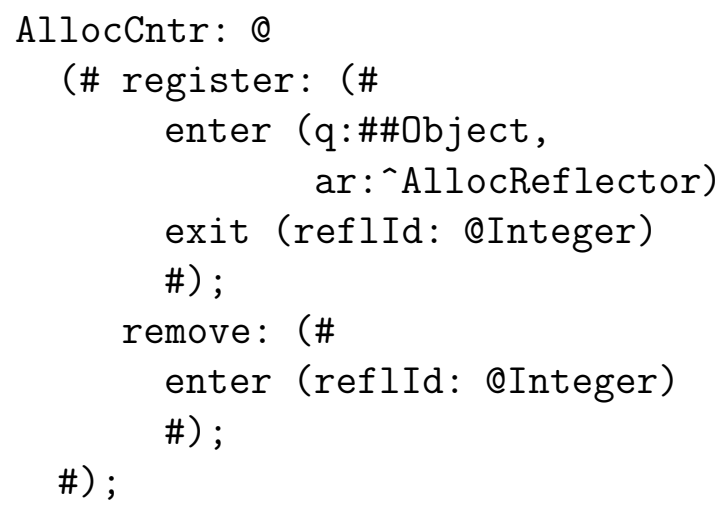

Figure 5: AllocCntr interface.

and a Car is created. The Vehicle creation only triggers VehicleAR.onAlloc, whereas the Car creation triggers VehicleAR. onAlloc and then CarAR. onAlloc, since Vehicle is a superpattern of Car. As a result of executing Figure 6,

\section{A: Vehicle \\ B: Vehicle Car}

is therefore printed to the screen.

The AllocCntr allocates the new object before any AllocReflector is called. Hence, reflectors cannot change the type or size of a newly created object, a limitation which avoids severe performance penalties. In particular, the size and type of new objects must be known at compile-time, to allow objects to be statically inlined in other objects and to support type-inference.

Implementation: AllocReflectors can be efficiently implemented by adding an extra word to the runtime class representation. Figure 7 illustrates the runtime data structures built in response to the registration of the two AllocReflectors from Figure 6.

The registration of VehicleAR installs a reference from the Vehicle class object to VehicleAR, and a reference from the Car class object to VehicleAR, since Car is a subclass of Vehicle, and since a more specific AllocReflector was not already installed for Car. The subsequent registration of CarAR overwrites the reference from the Car class object to VehicleAR, since the VehicleAR reference was "inherited" from the Vehicle class object. If multiple reflectors are registered with the same class, this will be represented as a linked list of reflectors.

Testing for an AllocReflector can now be implemented as a constant-time check for the presence of a reflector in the runtime class representation for the newly created object. However, this overhead will negatively affect nonreflective applications, and is therefore not acceptable. However, by exploit- 


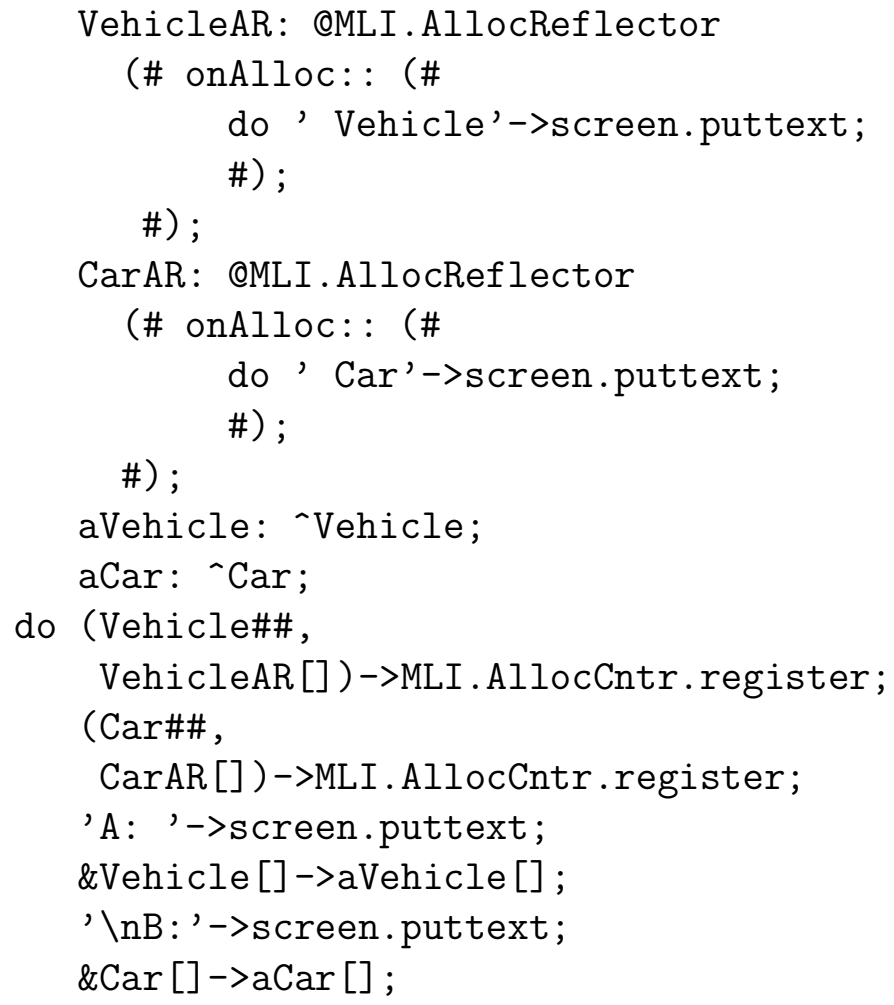

Figure 6: AllocReflector Example

ing the copying collection scheme used in the youngest object generation of the Mjølner BETA System, an implementation with no overhead on non-use can be achieved. In BETA, object creation is efficiently performed by bumping the topof-heap pointer with the instance size retrieved from the class object, followed by a check that no heap-overflow occurred. In MetaBETA, this implementation is unchanged, and thus no additional overhead is imposed. However, if an AllocReflector is installed in a class object, we arrange for the class object to return an instance-size large enough to force a heap-overflow. On heap-overflow, an extra check will determine whether it is actually time for a garbage-collection, or whether an AllocReflector is to be invoked.

\subsubsection{Object Execution}

MetaBETA allows programmer defined ExecReflector objects to be invoked on object execution events, so the default implementation can be specialized. An ExecReflector is defined as: 


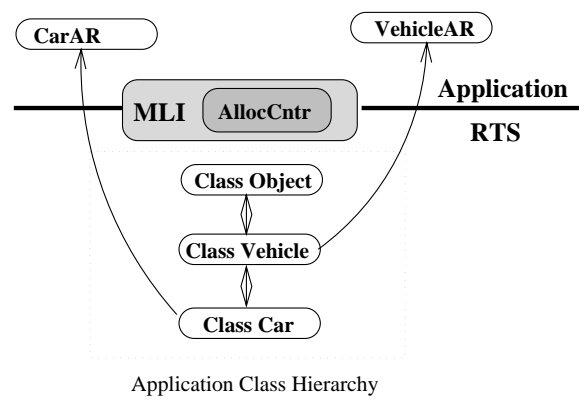

Figure 7: AllocCntr Runtime Representation.

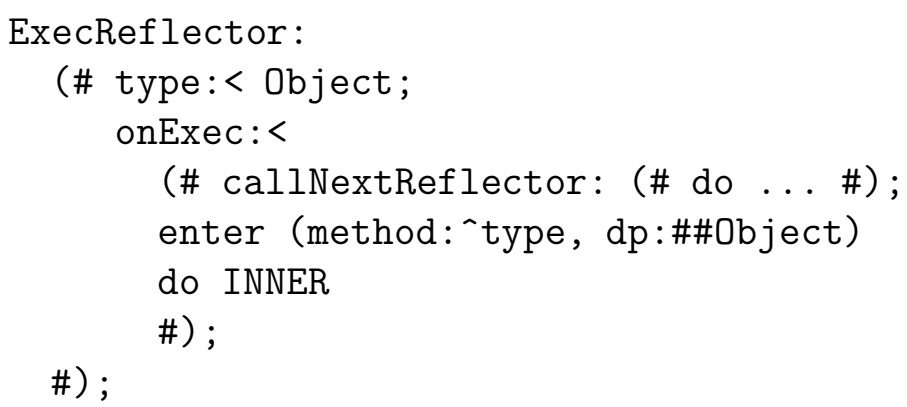

The ExecCntr metaobject is responsible for invoking the onExec virtual when specific do-parts are executed. To understand the exact ExecReflector semantics, we first take a closer look at BETA method execution.

The method call $(5,4)->a C a l c$.add- $>\operatorname{res}^{7}$ (from Figure 3 ) is executed in the following steps:

1. A new instance, method, of the aCalc.add pattern is created ${ }^{8}$. method is an ordinary BETA object playing the role of an activation record.

2. The actual input list, $(5,4)$, is evaluated and assigned to the formal enter list of method, thus assigning 5 to method.a and 4 to method.b.

3. The do-part of method is executed, assigning 9 to method.c.

4. The formal exit list, (c), of method is evaluated and assigned to the actual output list (res). Hence, the value of method.c is assigned to res.

Notification of ExecReflector objects embraces step 3 above. This allows the ExecReflector to query and change the value of attributes of the method object before and after its execution, and, if necessary, to actually execute the method object itself. Thus, the ExecCntr metaobject exposes three aspects to the

\footnotetext{
${ }^{7}$ Syntactic sugar for $(5,4)->$ \&aCalc add->res, \& being the object creation operator.

${ }^{8}$ If add had been a virtual pattern, the allocation step would include a table lookup to retrieve the exact binding of add.
} 
scrutiny of ExecReflectors: The state of the method object before execution, the actual execution of the method, and the state of the method object after execution.

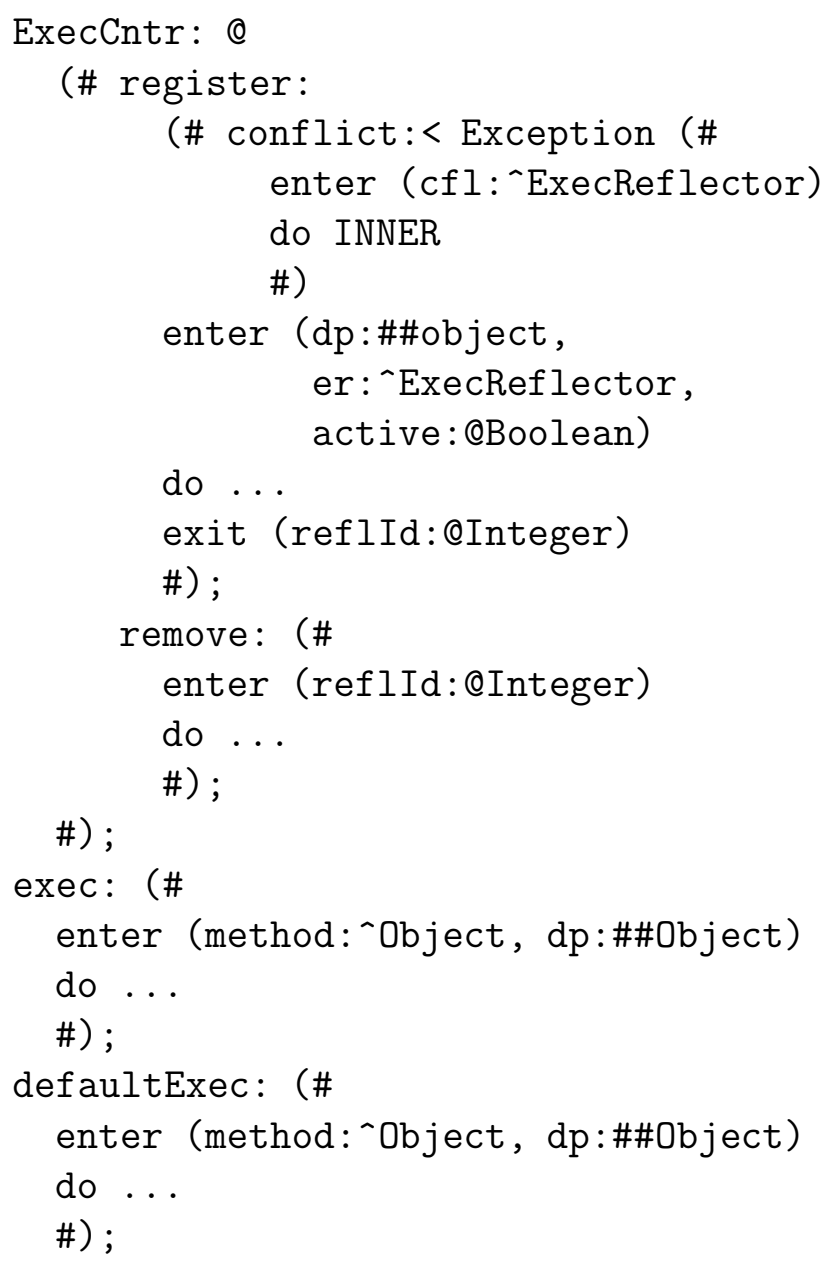

Figure 8: ExecCntr interface.

The ExecCntr interface is shown in Figure 8. To register an ExecReflector, metalevel code calls ExecCntr.register with a do-part specification dp, an ExecReflector parameter er, and a boolean parameter active. Then, whenever an object that qualifies to dp is about to execute the dp do-part, ExecCntr calls the er.onExec virtual with the method object and do-part to be executed as parameters.

The active parameter to ExecCntr.register reflects whether er handles the execution of method objects itself, or whether it simply monitors object execution events.

If several ExecReflectors apply, passive reflectors are executed in the same order as described for AllocReflector objects. An ExecReflector passes on 
control to the next reflector in the chain by calling callNextReflector. When all passive reflectors have been executed, callNextReflector passes on control to a possible active reflector. It is called last since it is responsible for actually executing the object. If no active reflector applies, the method object is automatically executed when the last passive ExecReflector calls callNextReflector. Usually there should be at most one active reflector applicable. If more than one is registered, the conflict exception will be raised at registration time. However, by catching and ignoring or explicitly sorting out conflicts, it is possible to register several active reflectors applying to the same object execution.

exec is a procedural interface to the object execution primitive. It executes the do-part of an object starting at a specified level of the specialization chain. Using exec to execute an object will trigger possible ExecReflector's installed for the object, whereas defaultExec will not. These methods can be used by active programmer-defined onExec methods to execute an object.

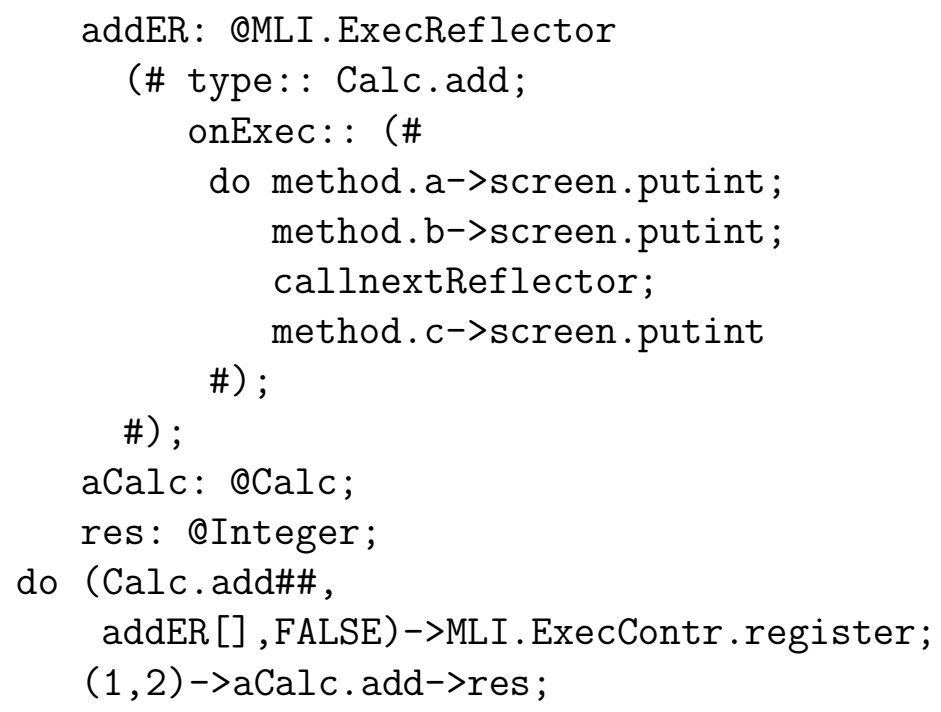

Figure 9: Using an ExecReflector.

Example 6 Assume that we want to monitor calls to the add method of Calc objects from Figure 3. To do so, an ExecReflector is associated with a dynamic pattern reference to Calc.add, as shown in Figure 9. Execution of Calc.add instances is then redirected into execution of addER. onExec, whose method parameter will refer the aCalc.add instance about to be executed. addER. onExec prints the parameters to the method call, and then calls callNextReflector to execute the method object. Notice how addER specializes the type virtual to $\mathrm{Calc}$. add, giving onExec static knowledge of the attributes of the method object.

The registration of addER shown in Figure 9 makes addER reflect on the execution of the add method of any Calc instance. Alternatively, if reflection is only 
wanted for a specific Calc instance, e.g., aCalc, then addER can been registered as:

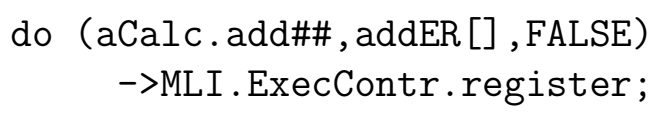

The difference is that the latter uses the aCalc.add\#\# qualification, whereas Figure 9 used the more general Calc.add\#\# qualification. Reflection happens on the same do-part, but the extension of aCalc.add is smaller than the extension of Calc.add, thereby restricting reflection to take place on a smaller set of object executions.

Example 7 The following code shows an ExecReflector that might be used to implement object distribution. Methods to be executed in a remote address space are packed and sent to the remote host for execution:

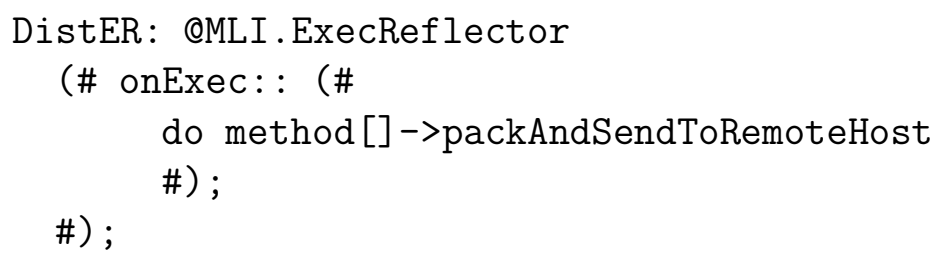

On the remote host, the method object would get unpacked, executed, (using exec or defaultExec), and finally sent back. DistER is an active ExecReflector, and the following code registers the aCalc. add method for remote execution:

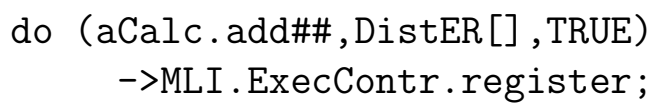

Implementation Execution reflection is implemented by runtime code modifications. Since patching does not occur until an ExecReflector is installed, and patches are undone when the ExecReflector is removed, there is no overhead for programs that do not use the mechanism.

Reconsider Example 6: It was demonstrated that we could either choose to reflect on the execution of all add methods, using the qualification Calc.add\#\#, or we could choose to reflect only on the execution of the add method of a specific Calc object, using the qualification aCalc.add\#\#. In any case, the code patch will be applied to the piece of machine-code that implements the add do-part. Thus, to reflect on aCalc.add\#\# executions, patch code must verify that the current execution does not correspond to the execution of the add method of a Calc instance different from aCalc, before invoking the installed reflector. As a result, reflection on the aCalc.add\#\# qualification will induce an overhead on all 
methods qualifying to Calc.add\#\#. Preliminary measurements in Section 5 will illustrate this point.

\subsubsection{Object Reclamation}

The GCCntr metaobject shown in Figure 10 allows MetaBETA programs to monitor the reclamation of specific objects. BETA is garbage-collected, so objects are never explicitly deallocated.

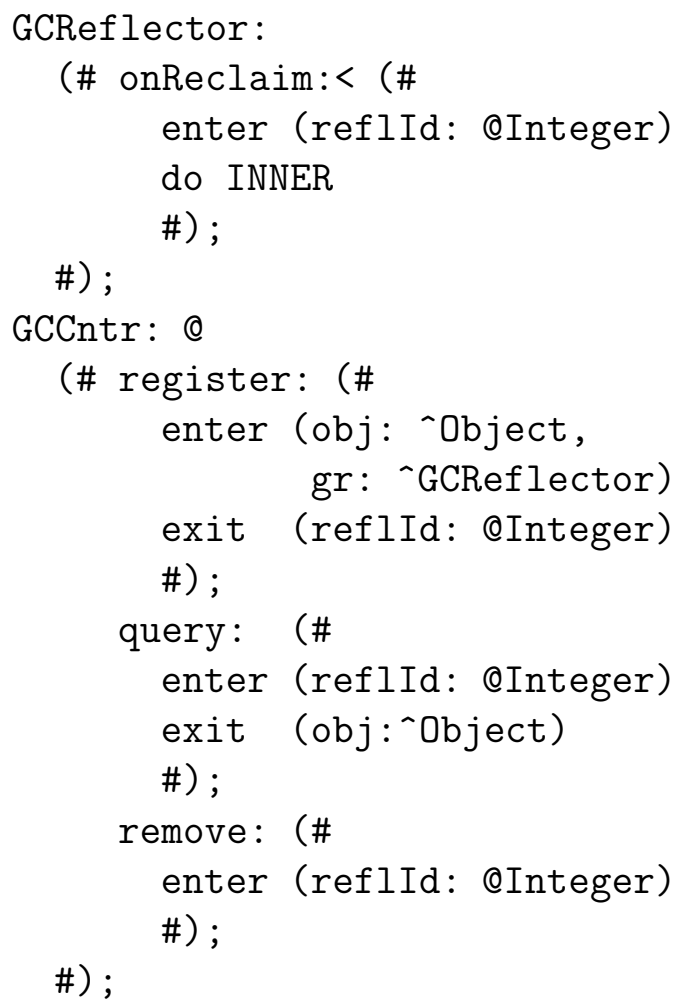

Figure 10: GCCntr interface.

To register a GCReflector, metalevel code calls GCCntr.register with an object parameter obj and a GCReflector parameter gr. Then, when obj becomes garbage, gr.onReclaim is called, and passed the reflId to identify the dead object. reflId is a unique receipt for the registration of a reflector, and can be used to remove a reflector or to obtain a direct reference to the object corresponding to reflId, using GCCntr. query. In this way, reflId can be seen as a weak object reference.

GCCntr does not allow garbage-collection of an object to be prevented, since this would introduce problems comparable to those of incremental and concurrent garbage-collection. Likewise, GC-tracing of all instances of some pattern is not 


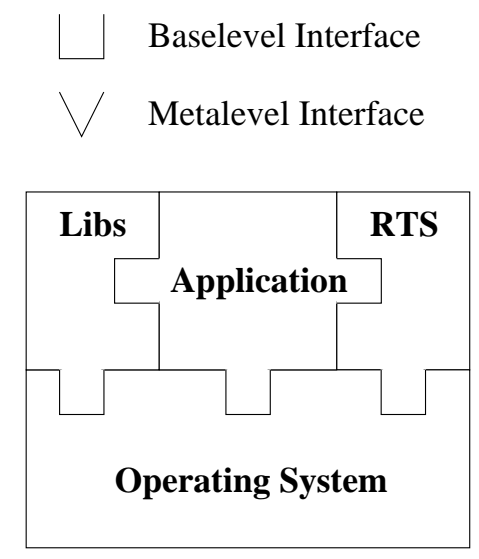

(a)

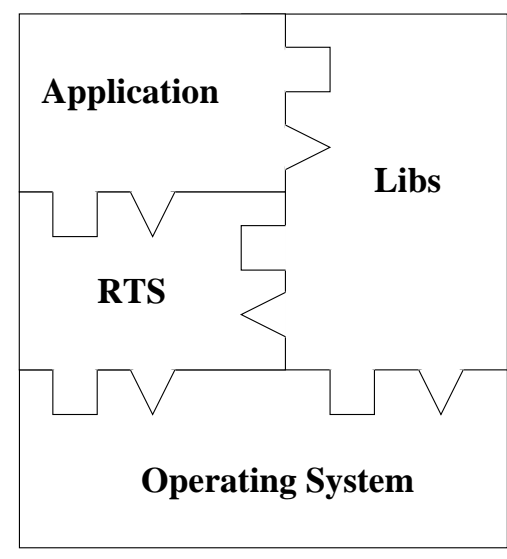

(b)

Figure 11: System Structures for: (a) a traditional compiled language, and (b) the metalevel architecture.

supported: In the case of a copying garbage-collector, that would require an expensive scan of all garbage after each collection.

Functionality corresponding to GCCntr already exists in the current BETA implementation, and is used by the source code debugger to track objects. However, the functionality is not available through a public interface.

\section{The Metalevel Architecture}

The implementation of a program with a runtime metalevel interface must maintain a causal connection to the metainformation at run-time. However, for most compiled languages, there is no specific component responsible for executing the code and maintaining the metainformation. The compiler generates machinecode to be executed directly by the processor.

The MetaBETA metalevel architecture is a runtime organization for compiled languages that supports a metalevel interface by making the runtime system responsible for code execution and metainformation maintenance. The organization is very similar to the implementation of most semi-interpreted and dynamically typed languages, such as CLOS [Kiczales et al. 91] and Smalltalk [Goldberg \& Robson 89].

\subsection{Runtime Organization}

We think of the runtime system as a virtual machine extending the underlying operating system. In the same way as binary programs may be considered data to be executed by the operating system, we can think of compiled programs as data to be executed by the runtime system. Conceptually, the runtime system is 
responsible for executing the compiled code. In practice, it hands the machinecode part of the compiled program to the underlying CPU for direct execution.

The virtual machine view of the runtime system induces a different system structure than for traditional compiled languages. The difference is shown in Figure 11. For a traditional compiled language, the application runs directly on top of the operating system, supported by the runtime system (RTS) and libraries. The runtime system, operating system, and libraries are viewed as black box implementations, i.e., they can only be accessed through baselevel interfaces. In the metalevel architecture (Figure 11b) the runtime system is the central component. It is responsible for running the application and providing metainformation to the application and libraries through its metalevel interface. The runtime system and libraries can also use a metalevel interface provided by the operating system [Kiczales \& Lamping 93].

The benefit of the virtual machine view is that it conceptually as well as in practice defines an entity to provide the metalevel interface for a compiled program.

\subsection{The Runtime System}

A central responsibility of the runtime system is to load and link compiled MetaBETA code. The compiler generates code to the MetaBETA runtime system, not to the operating system. A special code-format is used that contains the usual machine-code part, and also the metainformation needed by the metalevel interface.

Thus, the runtime system is the only executing program seen by the operating system. To execute a compiled MetaBETA program, the runtime system is started from the command-line with a parameter specifying which application it has to load and execute. This is in contrast to a traditional compiled language where the compiler generates executables for each application.

Since the runtime system is responsible for linking and loading code it can maintain and update the metainformation for a given program execution. Furthermore, it knows the code-layout in memory so runtime code-modification can be implemented efficiently.

\section{Status}

The implementation of MetaBETA is underway. Our strategy is to make prototype implementations of specific parts of the metalevel interface by extending the current Mjølner BETA implementation. This approach allows us to quickly implement and test parts of the MLI, and to verify the design on real metacode. Also, several implementation issues can be tested and verified independently. We 
are also working on a detailed design for the BETA virtual machine, i.e., the code-format, the loader, and the internal representation of metainformation.

\begin{tabular}{|c|r|r|r|r|}
\hline & & w/ & \multicolumn{2}{|c|}{ Overhead } \\
\cline { 3 - 5 } Pattern & Normal Reflector & Total & Patch \\
\hline \multicolumn{5}{|c|}{ Pattern Reflection } \\
\hline A & 1.04 & 3.25 & $212 \%$ & $16 \%$ \\
\hline B & 1.29 & 3.47 & $167 \%$ & $15 \%$ \\
\hline \multicolumn{5}{|c|}{ Object Reflection } \\
\hline objA & 0.29 & 2.43 & $738 \%$ & $19 \%$ \\
\hline objA $^{\prime}$ & 0.29 & 0.40 & - & $40 \%$ \\
\hline
\end{tabular}

Table 2: Reflector Overhead. Times are in $\mu$ secs and is measured on a $75 \mathrm{MHz}$ SparcStation 20.

A working prototype of the ExecReflector has been implemented with the main purpose of examining the overhead of inserting reflectors at runtime by patching binary code ${ }^{9}$. To determine the overhead, we have measured the overhead of reflecting on a specific pattern (Pattern Reflection), and on a specific instance of a pattern (Object Reflection). The results are shown in Table 2. The patterns A, B, and Refl are defined as follows:

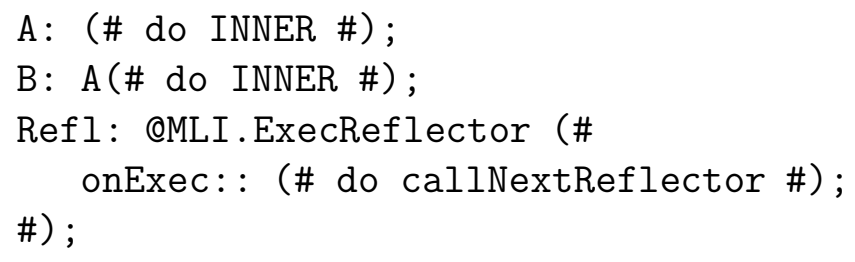

For pattern reflection we both measure the time to execute a top-level pattern A, and its subpattern B, because the BETA compiler generates a different call sequence for code called through INNER.

Most of the Total Overhead shown in Table 2 is the inherent overhead of creating and invoking the Refl.onExec method, which takes $1.73 \mu$ secs. Thus, the patch-code overhead is $T_{w / \text { reflector }}-\left(T_{\text {normal }}+T_{\text {Refl }}\right)$. It is shown in percent in the last column.

Reflection on execution of a single object requires that the patch code checks whether it actually applies to the current object execution. This overhead is measured under Object Reflection: objA and objA' are both instances of the A pattern, but only execution of objA is reflected upon. The time for executing an existing object is much faster than object creation and execution, because it does not require a new object to be allocated. Hence, the total overhead is much worse

\footnotetext{
${ }^{9}$ The prototype does not as yet support dynamic link and load of code.
} 
than for pattern reflection. Installing a reflector on a specific object introduces an invocation overhead of $0.11 \mu$ s on other objects of the same type.

We expect these preliminary numbers to be acceptable as compared to the overhead of, e.g., implementation of object distribution by other means. However, to verify this claim, future work includes the implementation of distributed BETA based on MetaBETA abstractions.

\section{Related Work}

The work presented in this paper is related to a number of previous research efforts in reflective language design and implementation. How they relate to MetaBETA is described in the following.

CLOS [Kiczales et al. 91] includes a comprehensive metalevel interface, (the CLOS MOP, or metaobject protocol), which allows the metalevel programmer to inspect and change several primitives of the programming language, including redefinition of slot access, multiple inheritance semantics, and replacement of metaclasses. The CLOS MOP greatly influenced the design of the introspective part of the MLI. However, the scope of the intercessory parts in BETA is on purpose much more limited, because we do not aim at changing fundamental language semantics such as inheritance order and scoping rules. Our focus is more on opening up the implementation of a few basic language primitives.

Also, BETA is statically typed, whereas CLOS is dynamically typed, leading to a considerably different style of metalevel interface. Most notably, the BETA MLI allows, but does not force, the metalevel programmer to express static typing constraints, thereby leading to more readable and possibly more efficient metalevel code. ABCL/R2 [Masuhara et al. 92], AL-1/D [Okamura et al. 93], and SELF [Hölzle \& Ungar 94] are further examples of dynamically typed programming languages with reflectional capabilities. As with CLOS, the most notable difference between the metalevel interfaces of these languages and MetaBETA, is the issue of static versus dynamic typing.

Open C++'s [Chiba 93] (version 1) concept of reflect methods was the direct inspiration for the reflector mechanism used in MetaBETA to reify basic language primitives. A reflector in MetaBETA controls the implementation of a specific language primitive, which is similar to the CodA [McAffer 95] system for Smalltalk.

The newest version of Open C++ [Chiba 95] implements a compile-time metaobject protocol, with metalevel code controlling the compilation of baselevel code. A similar approach is used in the START system [Kirby et al. 94], implemented for the Napier88 language [Morrison et al. 93]. These systems offer compile-time reflection, and thus assumes that the metalevel is statically known. In comparison, MetaBETA is entirely dynamic, with reflective hooks inserted at run-time, i.e., MetaBETA offers later binding than systems based on compile-time reflec- 
tion. ABCL/R3 [Masuhara et al. 95] may be seen as a combination of the two approaches: Partial evaluation attempts to compile away interpretation of the metalevel, whereas different code-versions support runtime change of metaobjects.

The $\mathrm{C}++$ concept of pointers to members may be considered a restricted version of attribute references, which the MetaBETA MLI is based on. However, the absence of runtime type information, run-time type checking, and restriction to function members renders $\mathrm{C}++$ pointers to members less powerful than attribute references.

\section{Conclusion}

This paper desribed an extension of the statically-typed and compiled language BETA with a metalevel interface. Our metalevel interface is based on first-class attributes and classes, which allows metalevel computations to be easily integrated into the existing language without circumventing or changing the existing type-system.

Our metalevel interface is a runtime entity giving a running program access to the representation of objects. The use of the metalevel interface does not require access to source code. This provides a large flexibility that allows metalevel code to be applied to classes written before the metalevel code itself.

Previous metalevel interfaces for statically typed languages have either circumvented the language type-system, or has been based on a compile-time MLI. With the introduction of attribute references, MetaBETA provides a both runtime based and type-safe metalevel interface for statically typed languages.

\section{Acknowledgements}

We would like to thank Ole Lehrmann Madsen and Jørgen Lindskov Knudsen who read earlier versions of this paper and provided many valuable comments.

\section{References}

[Arnold \& Gosling 96] K. Arnold and J. Gosling. The Java Programming Language. Addison-Wesley Publishing Company, Reading, MA, March 1996.

[Brandt \& Knudsen 96] S. Brandt and J. L. Knudsen. Patterns and Qualifications in BETA: A Case for Generalization. In Proceedings of the Ninth European Conference on Object-Oriented Programming (ECOOP), July 1996. 
[Brandt \& Madsen 94] S. Brandt and O. L. Madsen. Object-Oriented Distributed Programming in BETA. In R. Guerraoui, O. Nierstrasz, and M. Riveill, editors, Lecture Notes in Computer Science 791, pages 185 - 212. Springer-Verlag, January 1994.

[Brandt 94] S. Brandt. Implementing Shared and Persistent Objects in BETA Progress Report. Technical report, Department of Computer Science, University of Aarhus, May 1994.

[Chiba 93] S. Chiba. Designing an Extensible Distributed Language with a MetaLevel Architechture. In O. Nierstrasz, editor, Proceddings of the 7th European Conference on Object-Oriented Programming, volume 707 of LNCS, pages 482 - 501. Springer-Verlag, July 1993.

[Chiba 95] S. Chiba. A Metaobject Protocol for C++. In Proceedings of the 10th Conference on Object-Oriented Programming Systems, Languages, and Applications (OOPSLA), October 1995.

[Goldberg \& Robson 89] A. Goldberg and D. Robson. Smalltalk-80. The Language. Addison-Wesley, Reading, MA, 1989.

[Grønbæk et al. 94] K. Grønbæk, J. Hem, O. Madsen, and L. Sloth. Hypermedia Systems: A Dexter-Based Architecture. Communications of the ACM, 37(2):64 - 74, February 1994.

[Hölzle \& Ungar 94] U. Hölzle and D. Ungar. A Third Generation Self Implementation: Reconciling Responsiveness with Performance. In Proceedings of the 9th Conference on Object-Oriented Programming Systems, Languages, and Applications (OOPSLA), pages 229 - 243, October 1994.

[Kiczales \& Lamping 93] G. Kiczales and J. Lamping. Operating Systems: Why Object-Oriented? In Proceedings of International Workshop on ObjectOrientation in Operating Systems (IWOOS), 1993.

[Kiczales 92] G. Kiczales. Towards a new model of Abstraction in Software Engineering. In A. Yonezawa and B. C. Smith, editors, Proceedings of International Workshop on Reflection and Meta-level Architecture (IMSA), pages 1-11, November 1992.

[Kiczales et al. 91] G. Kiczales, J. Rivieres, and D. Bobrow. The Art of the Metaobject Protocol. MIT Press, 1991.

[Kirby et al. 94] G. Kirby, R. Connor, and R. Morrison. START: A Linguistic Reflection Tool Using Hyper-Program Technology. In Proceedings of the 6th International Workshop on Persistent Object Systems, pages 346 365, September 1994. 
[Madsen et al. 93] O. L. Madsen, B. Møller-Pedersen, and K. Nygaard. ObjectOriented Programming in the BETA Programming Language. Addison Wesley, Reading, MA, 1993.

[Malhotra 93] J. Malhotra. Dynamic Extensibility in a Statically-Compiled Object-Oriented Language. In Proceedings of the International Symposium on Object Technologies for Advanced Software (ISOTAS), Kanazawa, Japan, November 1993.

[Masuhara et al. 92] H. Masuhara, S. Matsuoka, T. Watanabe, and A. Yonezawa. Object-Oriented Concurrent Reflective Languages can be Implemented Efficiently. In A. Paepcke, editor, Proccedings of the "th Conference on Object-Oriented Programming Systems, Languages, and Applications (OOPSLA), pages 127 - 144, October 1992.

[Masuhara et al. 95] H. Masuhara, S. Matsuoka, K. Asai, and A. Yonezawa. Compiling Away the Meta-Level in Object-Oriented Concurrent Reflective Languages Using Partial Evaluation. In Proceedings of the 10th Conference on Object-Oriented Programming Systems, Languages, and Applications (OOPSLA), October 1995.

[McAffer 95] J. McAffer. Meta-Level Programming with CodA. In Proceedings of the 9th European Conference on Object-Oriented Programming (ECOOP), August 1995.

[Meyer 92] B. Meyer. Eiffel, The Language. Prentice Hall, 1992.

[Morrison et al. 93] R. Morrison, A. L. Brown, R. C. H. Connor, Q. I. Cutts, A. Dearly, G. N. C. Kirby, and D. S. Munro. The Napier88 Reference Manual (Release 2.0). Technical Report CS/93/15, University of St Andrews, 1993.

[Okamura et al. 93] H. Okamura, Y. Ishikawa, and M. Tokoro. Metalevel Decomposition in AL-1/D. In Proceedings of the International Symposium on Object Technologies for Advanced Software (ISOTAS), November 1993. 